\title{
Evaluation of the EMPAR study population on the basis of metabolic phenotypes of selected pharmacogenes
}

\author{
Jochen Fracowiak ${ }^{1,6}$, Tatjana Huebner ${ }^{1,6}{ }^{凶}$, Steffen Heß ${ }^{1}$, Christoph Roethlein $^{2}$, Daria Langner ${ }^{3}$, Udo Schneider ${ }^{3}$, Felix Falkenberg $^{3}$,
} Catharina Scholl ${ }^{1}$, Roland Linder ${ }^{3}$, Julia Stingl $\mathbb{D}^{4}{ }^{4}$, Britta Haenisch ${ }^{1,2,5}$ and Michael Steffens $\mathbb{D}^{1}$

(c) The Author(s) 2022

The impact of genetic variability of pharmacogenes as a possible risk factor for adverse drug reactions is elucidated in the EMPAR (Einfluss metabolischer Profile auf die Arzneimitteltherapiesicherheit in der Routineversorgung/English: influence of metabolic profiles on the safety of drug therapy in routine care) study. EMPAR evaluates possible associations of pharmacogenetically predicted metabolic profiles relevant for the metabolism of frequently prescribed cardiovascular drugs. Based on a German study population of 10,748 participants providing access to healthcare claims data and DNA samples for pharmacogenetic assessment, first analyses were performed and evaluated. The aim of this first evaluation was the characterization of the study population with regard to general parameters such as age, gender, comorbidity, and polypharmacy at baseline (baseline year) as well as important combinations of cardiovascular drugs with relevant genetic variants and predicted metabolic phenotypes. The study was registered in the German Clinical Trials Register (DRKS) on July 6, 2018 (DRKS00013909).

The Pharmacogenomics Journal (2022) 22:136-144; https://doi.org/10.1038/s41397-022-00268-6

\section{INTRODUCTION}

Preemptive pharmacogenetic testing in routine care can identify risk factors for adverse drug reactions (ADRs) related to genetic variability and thus decrease the risk of ADRs that cause high costs and jeopardize the health of patients. About $5-15 \%$ of hospitalizations are linked to ADRs [1, 2]. However, the implementation of preemptive PGx testing for preventive measures in clinical laboratories faces many barriers, especially the lack of data from studies with large study populations, the complexity of PGx analysis and reimbursement policies [3].

Routine care records provide useful data on the utilization of health services and health expenditure. For this cooperation project, such relevant routine care records are provided after the successful recruitment of Techniker Krankenkasse (TK) insurants by the TK, a large statutory health insurance company with about 10.8 million insurants in Germany [4]. TK insurants recruited, furthermore, provided genetic samples and permission for assessment of their genetic data on pharmacogenes.

One major group of pharmacogenes with genetic variability affecting drug response of several lead substances is the Cytochrome P450 protein family that is responsible for about $75 \%$ of all phase I drug reactions in drug metabolism [5]. Thus, cytochromes such as CYP2C9, CYP2C19, CYP3A4, and CYP3A5 are considered in the study evaluations. Furthermore, genetic variants of the vitamin $\mathrm{K}$ epoxide reductase complex subunit 1 (VKORC1) involve a risk for serious ADRs such as bleeding events in drug therapy with phenprocoumon and warfarin [6] and variants of transporters like Solute Carrier Organic Anion Transporter Family Member 1B1 (SLCO1B1) and ATP Binding Cassette Subfamily B
Member $1(A B C B 1)$, which affect the transport of statins are of interest [7-9]. Several databases are in place to support pharmacogenetic assessments, such as the Single Nucleotide Polymorphism Database (dbSNP) for collecting and archiving data on genome variations and frequencies from large genome studies [10]. Research consortia such as PharmVar [11] and databases such as The Pharmacogenomics Knowledge Base (PharmGKB) [12] collect, review, and provide valid information on known variations of pharmacogenes, which can be further used to deduce their metabolic phenotype. Furthermore, PharmGKB provides information on gene-drug relationships that are clinically actionable and therefore potentially require variant-specific clinical action [13]. In this evaluation, pharmacogenetic variants of the high PharmGKB Clinical Annotation Levels of Evidence $1 \mathrm{~A}$ or $1 \mathrm{~B}$, therefore, are referred to as actionable variants [14]. Here, we present the first data on pharmacogenetic analyses in the EMPAR (Einfluss metabolischer Profile auf die Arzneimitteltherapiesicherheit in der Routineversorgung/English: influence of metabolic profiles on the safety of drug therapy in routine care) study involving the sofar largest collection of pharmacogenetic profiles with health insurance data in Germany. In this first evaluation, a focus is set on variants of genes with an impact on absorption, distribution, metabolism, and elimination of lead substances relevant for cardiovascular conditions. Cardiovascular diseases are events of elevated prevalence among elderly people in Germany and one of the leading causes of mortality in developed countries $[5,6]$. Therefore, analyses were focused on the anticoagulant/ antiplatelet and the cholesterol-lowering drug collective of the EMPAR study population. Furthermore, routine care records of TK

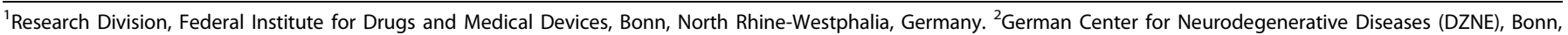

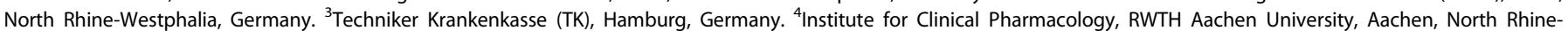

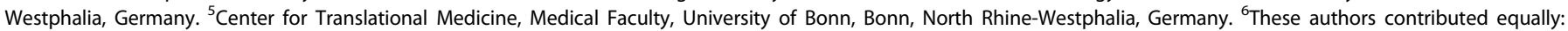
Jochen Fracowiak, Tatjana Huebner. ${ }^{凶}$ email: Tatjana.Huebner@bfarm-research.de 
insurants such as age, gender, medication, and diagnoses in the year prior to prescription of the main medication of study interest were analyzed.

\section{METHODS \\ Study design and recruitment}

EMPAR recruitment was initiated in 2018 in Germany with a target sample size of about 10,000 adult study participants insured by TK. The present study population comprises three patient collectives. The two cardiovascular drug collectives (anticoagulant/antiplatelet and cholesterol-lowering drug collective) were of major interest for essential evaluations in this study. The International Classification of Diseases (ICD-10) Y57.9! collective serves for screening for new relevant single-nucleotide polymorphisms (SNPs) associated with ADRs in future investigations and was only addressed in this first evaluation with regard to general characteristics such as age, gender, polypharmacy, morbidity, and actionable variants. Further details on the study design were published by Huebner et al. and in the German Clinical Trials Register [15, 16]. For an additional recruitment phase in 2020, the period of initial prescription for the anticoagulant/antiplatelet and cholesterol-lowering drug collective was extended until the end of 2017 in order to reach the target sample size. Recruited participants provided written informed consent and sent a questionnaire and buccal swab samples to the Deutsche Knochenmarkspenderdatei Life Science Lab GmbH (Dresden, Germany) for sample collection and DNA extraction. DNA samples were genotyped via the pharmacogenomics services of Agena Bioscience Inc. (Hamburg, Germany) for the application of the iPLEX ${ }^{\oplus}$ PGx 74 and the VeriDose CYP2D6 CNV panel [17].

\section{Data quality control}

Genotype data of 67 markers, covering 19 genes [18], were quality controlled by computing marker call rates and minor allele frequencies (MAF). The latter were compared with data of the Caucasian population obtained from dbSNP. Allele frequencies were calculated by PLINK version 1.9 and tested for Hardy-Weinberg equilibrium (HWE) [19]. Due to data policy reasons, rs7412 and rs429358 (APOE) were excluded from analyses.

\section{Evaluations of collective characteristics}

Investigated parameters such as gender, age, diagnoses based on ICD-10 system, and medication were obtained from anonymized TK healthcare claims data. A time window of 1 year before individual prescription of the main medication was set for evaluations of population characteristics at baseline of the anticoagulant/antiplatelet and cholesterol-lowering drug collective. The Y57.9!-diagnosis collective was investigated up to 1 year before the first appearance of a Y57.9 or Y57.9!-diagnosis entry.

For an initial study population and collective description, ICD-10 entries of the individual baseline year were taken into account. Long-term polypharmacy was defined by a prescription frequency of $\geq 5$ medications, observed in a consecutive sequence of four quarters (baseline year). For comorbidity and polypharmacy assessment, stratified pharmacoepidemiological analyses of the three study collectives (cholesterol reducer, anticoagulant/antiplatelet, and Y57.9! diagnosis) were performed by age (young: <36; moderate: 36-60; old: >60) and gender (male, female). Comorbidity was calculated according to Elixhauser, implemented in the R-package comorbidity (version 0.5.3) [20]. All statistics for collective characterization were carried out using R 3.6.3 and Python 3.

\section{Evaluations of pharmacogenetic data}

PGx markers of study interest (Supplement 1 - Table 1) were used for further evaluations with regard to pharmacogenetic data and associated main medication of study participants. Pharmacogenes covered by single markers only (VKORC1, SLCO1B1) were directly translated into their corresponding star allele nomenclature. Pharmacogenes covered by more than one marker were firstly computational phased to haplotypes by applying PLINK's EM-algorithm and subsequently combined to diplotypes. The resulting diplotypes were translated into the star allele nomenclature, according to PharmGKB and scientific literature $[12,19]$. Corresponding metabolic phenotypes were determined based on the PhamGKB Diplotype-Phenotype translation tables. In case of CYP2D6, diplotypes were computed by additionally considering the gene copy number data and non-functional hybrid allele information $\left({ }^{*} 36,{ }^{*} 13\right.$, and ${ }^{*} 68$ ) (Agena Bioscience VeriDose CYP2D6 CNV Panel). Phenotypes were derived by using the activity scoring system, according to the CYP2D6 consensus definition, published by Caudle et al. [21]. All computed phenotypes primarily relevant for the cardiovascular drug metabolism or transport were compared with the corresponding marker frequencies in dbSNP and to frequencies reported in similar studies.

In this first assessment, the EMPAR study population was analyzed for allele distributions of actionable variants (variants of PharmGKB evidence level 1A or 1B [14]) relevant for the response to therapy with anticoagulants, antiplatelets or cholesterol reducers of study interest (main medication). This criterion was met by 13 markers (Supplement 2, Actionable variants). The identified actionable markers and the according cardiovascular indications were applied for filtering and assessment of allele distributions.

For further analyses of the cardiovascular drug collectives, also variants with lower evidence levels were considered (Supplement 1 - Table 1). For the anticoagulant/antiplatelet and cholesterol-lowering drug collective, respective pharmacogenes that could interact and thus may increase the impact on drug response were analyzed pairwise with regard to the occurrence and frequencies of diplotype combinations. Furthermore, diplotypes of six combinations of CYP3A4, CYP3A5, SLCO1B1, and $A B C B 1$ were analyzed. In phenotype analyses, frequencies of extreme phenotypes (ultrarapid or poor function) in combination with the affected prescribed main medication were assessed for each collective. All statistics for the evaluations of pharmacogenetic data were carried out by using Python 3 .

\section{RESULTS}

\section{Recruitment}

In total, 54,989 TK insurants were invited to participate in 2018. In 2020, the TK invited further 19,991 insurants with an initial prescription of anticoagulants/antiplatelets and cholesterol reducers in 2016-2017 to participate. About $16.7 \%$ of the eligible TK insurants consented to be included in the EMPAR study. About 11,026 (88.27\%) of those who sent in their written informed consent provided a DNA sample for pharmacogenetic genotyping. In total, data on relevant pharmacogenetic SNPs and copy number variations could be obtained from 10,791 participants. However, genotype data of 10,788 participants were suitable for further analyses according to data quality parameters (Fig. 1).

\section{Data quality control}

An overall marker call rate of $98.4 \%$ was calculated (Supplement 1 - Fig. 1). Moreover, 49 markers were tested to be in HWE and 18 significantly deviated from HWE (Supplement 1 - Fig. 2). The calculated MAFs in the EMPAR population showed no conspicuous deviation from the MAFs of the respective markers reported in dbSNP for the Caucasian European (CEU) population (Supplement 1 - Fig. 3). Three participants were dropped from the study due to low marker call rates. 
Recruitment 2018-2019

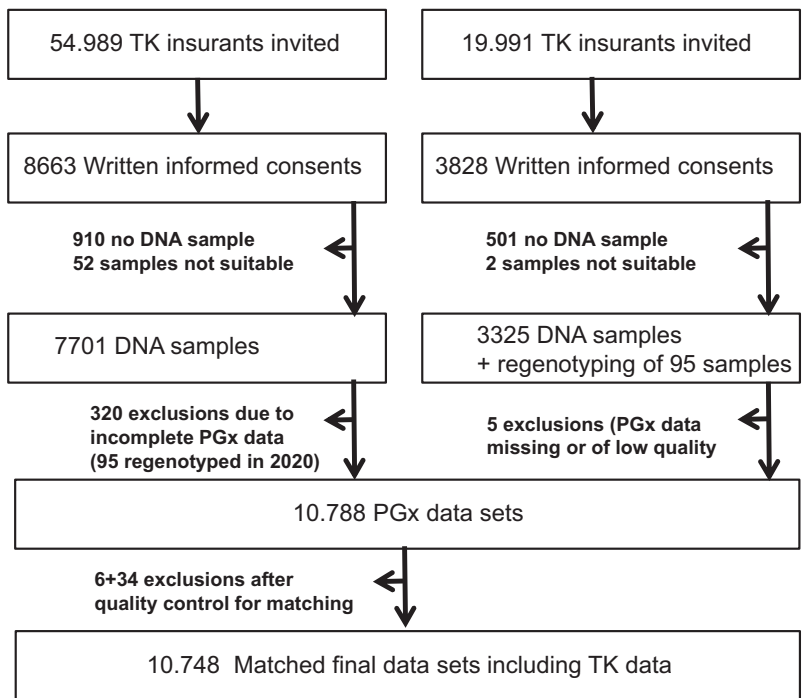

Fig. 1 Recruitment scheme of the EMPAR study in 2018-2020. Dropouts occurred in the provision of suitable DNA samples $(11.1 \%$ in $2018-2019$ and $13.1 \%$ in 2020), due to insufficient quality or a lack of genotype data $(2.2 \%)$ and due to insufficient quality of healthcare claims data for matching (0.4\%). PGx pharmacogenetic.

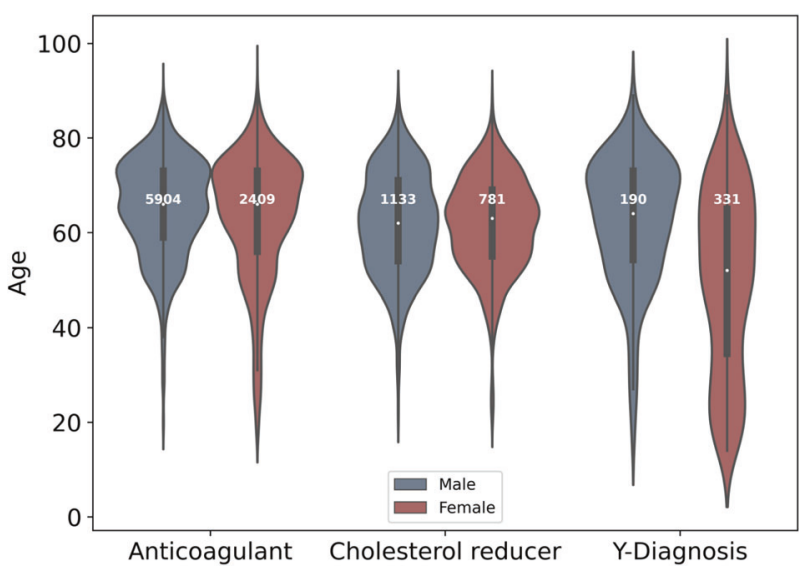

Fig. 2 Age and sex evaluation. Age distributions of male and female participants in each study collective of the complete study population (anticoagulant: anticoagulant/antiplatelet collective, cholesterol reducer: cholesterol-lowering drug collective, Y-diagnosis: Y57.9! diagnosis collective) at baseline.

\section{Study population characteristics}

Genotype data available from 10,788 participants were merged with the according healthcare claims data. Thereby, data records of six participants did not meet the necessary quality criteria in terms of ICD-10 code entry Y57.9! for further analyses. These participants were excluded from the study. Furthermore, subsequently, 34 participants of the cholesterol-lowering drug collective with prescriptions of combination products that include statins in the baseline year were excluded due to the according exclusion criterion for this collective (13.9\% overall dropout). In sum, healthcare claims data and phenotype data of 10,748 participants were successfully merged (Fig. 1). The study population comprises 8313 (77.3\%) participants of the anticoagulant/antiplatelet collective, 1914 (17.8\%) participants of the cholesterol-lowering drug collective and 521 (4.8\%) participants of the Y57.9!-diagnosis collective (Fig. 2).

In the evaluation of general collective characteristics, a stratified analysis of basal cohort morbidity by age (young: <36;

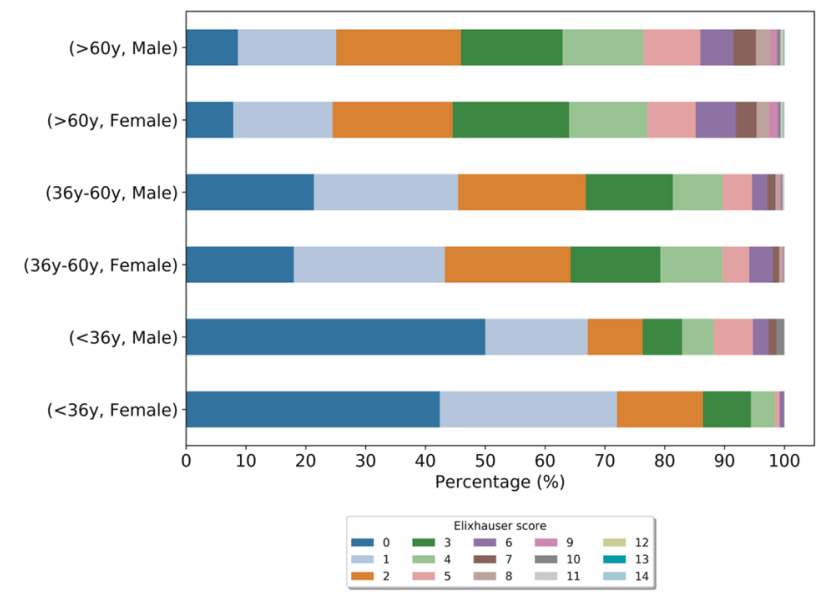

Fig. 3 Elixhauser comorbidity score distributions (in \%). All diagnosis codes (ICD-10) of the individual baseline year were taken into account. The score distribution reflects the comorbidity status of the complete study population in the baseline year grouped by age and gender.

moderate: 36-60; old: $>60$ ) and gender among all collectives (Supplement 3) elucidated an increasing number of diagnoses with age. This general tendency seems to be supported by the comorbidity distributions (Fig. 3) representing the mortality prediction in terms of the Elixhauser score that was used to assess the health situation before prescription of the main medication. Clear tendencies of higher risk scores from young to old participants were observed with marginal differences between males and females. Among the three collectives, the median ages of male and female participants in the baseline year were all above 60 years $(64.73$ years on average $(\sigma=11.78)$ ), except in the Y57.9!-diagnosis collective with a median age of 52 years for female participants.

The comorbidity analysis of the complete study population revealed 10,227 (93.9\%) participants with outpatient and inpatient ICD-10 entry in the individual baseline year. A total of 6226 different outpatient and inpatient ICD-10 diagnoses were observed. Overall, essential (primary) hypertension (I10.90), which is an important risk factor for different cardiovascular conditions [22], was the most frequent diagnosis in the mid and old age groups of all cohorts of the study population. Further frequently detected diagnoses relevant for cardiovascular conditions were disorders of lipoprotein metabolism and other lipidemias (e.g., E78.0, E78.5), benign essential hypertension (I10.00) and diabetes mellitus, type 2 (E11.90) (Supplement 3).

An investigation in the period of 1 year prior to prescription of the main medication (anticoagulant collective and cholesterollowering drug collective) or prior to Y57.9!-diagnosis (Y57.9!diagnosis collective) on the number of concomitant medication prescribed in the same quarter revealed that $9746(90.7 \%)$ participants obtained at least one prescription in the individual baseline year and 3635 (33.8\%) were identified with $\geq 5$ prescriptions in at least one quarter of the baseline year. A stratified assessment between concomitant medication, age groups, and collectives illustrates the highest median prescription number for the old age groups ( $>60$ years). Thereby, the highest counts of concomitant prescriptions in the baseline year were identified for the Y57.9!-diagnosis collective (Median: 7 prescriptions). In the cholesterol reducer collective, a median of 4 prescriptions and in the anticoagulant collective, a median of 5 prescriptions was identified in the old age group (Fig. 4). On average, $6.9(\sigma=5.53)$ different medications were counted per person. An analysis of long-term polypharmacy in the EMPAR population showed that $21 \%$ of participants had prescriptions of at least two medications within each quarter over the baseline year and about 242 (2.3\% of 


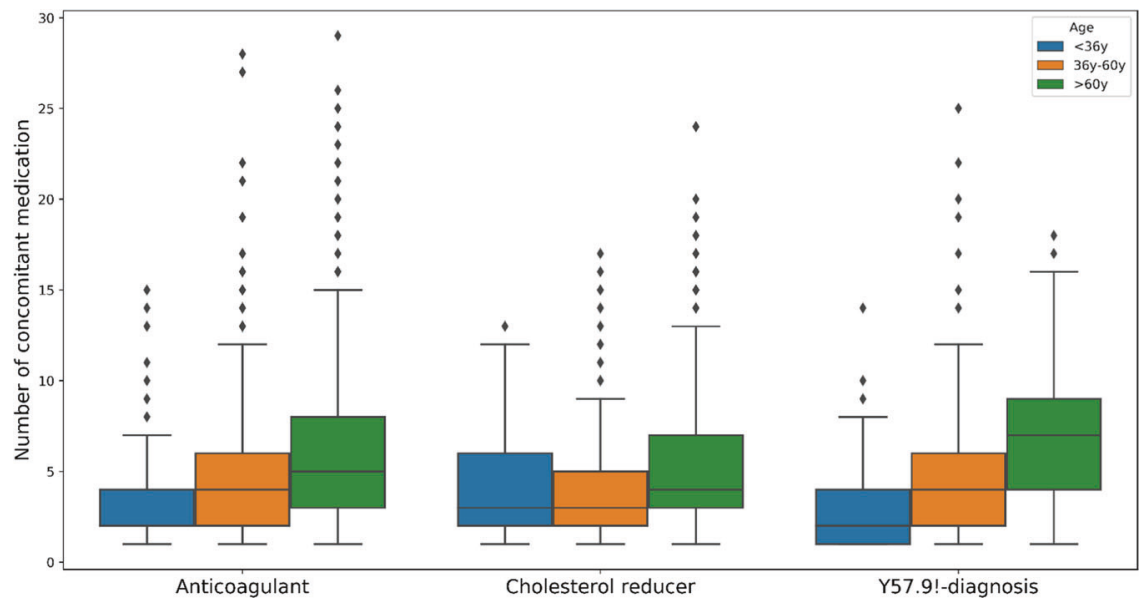

Fig. 4 Abundance of concomitant medication prescribed during the individual baseline year grouped by age and collective. Anticoagulant: anticoagulant/antiplatelet.

study participants) were affected by long-term polypharmacy at baseline (Table 1).

In the study population, a total of 113,203 prescriptions were counted within the baseline year, thereby also prescriptions of antagonists for CYP2C9 (11.03\%), CYP2C19 (8.79\%), VKORC1 (0.21\%), SLCO1B1 (21.92\%), ABCB1 (31.35\%), CYP3A4 (13.33\%), and CYP3A5 (0.04\%) were identified. In addition, a high percentage of participants within the cardiovascular drug collectives was identified with relevant prescriptions of inhibitors and antagonists that have a functional effect on the cytochromes, catalytic subunits, and transporters under study (Table 2). Especially, for clopidrogrel, simvastatin, and phenprocoumon, clinical annotations of a high level of evidence with regard to drug-gene pairs such as clopidrogrel-CYP2C19, simvastatin-SLCO1B1 and phenprocoumon-VKORC1 are present (Supplement 2, Medication). Clopidrogrel, simvastatin, and phenprocoumon were identified among the most frequently prescribed main medication in the EMPAR study population (Supplement 1 - Fig. 4). Therefore, they were of special interest concerning pharmacogenetic assessments and may also provide a sufficient sample size for future pharmacoeconomic assessments.

\section{Pharmacogenetic characteristics}

The analysis of the actionable variants reflected that $95.5 \%$ of the participants of the EMPAR study population were carrier of at least one actionable marker (Fig. 5). Seven of the 13 analyzed markers were monomorphic and corresponded to the wild type (Fig. 5A). Pharmacogenes primarily relevant (Supplement 1 - Table 1) with regard to the considered cardiovascular drugs were analyzed on diplotype (star nomenclature) and metabolic phenotype level. All metabolic phenotype distributions were comparable to reference (PharmGKB) or other study data in terms of the metabolic profile frequencies (Supplement 1 - Figs. 5-7). However, differences in metabolic profile distributions were detected as expected, especially for CYP2D6 as a result of the changed phenotypescoring algorithm according to the inclusion of VeriDose CYP2D6 CNV panel data.

Pairwise analysis of diplotypes of CYP2C19, CYP2C9, ABCB1, $V K O R C 1$ concerning the combination frequencies in the anticoagulant collective (Supplement 1 - Figs. 8 and 9) showed that relevant homozygous combinations that deviate from the normal function were rarely present.

Similar results could be observed in the cholesterol reducer collective for a pairwise analysis of selected combinations of CYP3A4, CYP3A5, SLCO1B1, and ABCB1 (Supplement 1 - Fig. 10). Further collective analysis was performed considering extreme
Table 1. Participants with prescribed medication in a consecutive sequence of four quarters at baseline (baseline year).

\begin{tabular}{lcr} 
Number of medication & Number of participants & $\%$ \\
\hline 1 & 2155 & 20.05 \\
\hline 2 & 1166 & 10.85 \\
\hline 3 & 571 & 5.31 \\
\hline 4 & 279 & 2.60 \\
\hline 5 & 135 & 1.26 \\
\hline 6 & 67 & 0.62 \\
\hline 7 & 23 & 0.21 \\
\hline 8 & 10 & 0.09 \\
9 & 6 & 0.06 \\
\hline 10 & 1 & 0.01
\end{tabular}

phenotypes (ultrarapid or poor function) (Table 3). Thereby, the focus in this first survey was on drug-gene combinations with sufficient evidence of impact according to PharmGKB clinical annotations (1A-3, Supplement 2, Medication) [23]. In the calculation for the anticoagulant/antiplatelet collective, $41.6 \%$ of participants of this subgroup were identified with at least one extreme phenotype of CYP2C9, CYP2C19, VKORC1, or ABCB1 in combination with prescriptions of anticoagulants or antiplatelet medication. In the large anticoagulant/antiplatelet collective clinically important drug-gene pair fractions were identified for clopidogrel or clopidogrel/acetylsalicylic acid + CYP2C19 poor metabolizer (PM)/ultrarapid metabolizer (UM) (2.1\%) with evidence level $1 \mathrm{~A}$ and phenprocoumon + VKORC1 rs9923231 TT (2.6\% with poor function) with The Clinical Pharmacogenetics Implementation Consortium (CPIC ${ }^{\oplus}$ ) evidence level 1B (Supplement 2). An analog approach was applied for all participants of the cholesterol reducer collective. Thereby, 1699 of 1914 participants $(88.8 \%)$ were identified with an extreme phenotype of CYP3A4, CYP3A5, SLCO1B1 or in case of $A B C B 1$ being homozygous alternating (rs1045642_GG). Only $0.8 \%$ of participants of the cholesterol-lowering drug collective were identified with the drug-gene combination simvastatin + SLCO1B1 PM (Table 3), the highest evidence-based drug-gene combination according to CPIC (1A). The drug-gene combination simvastatin + rs1045642_GG $(A B C B 1)$ with CPIC evidence level $2 \mathrm{~A}$ occurred in $10.1 \%$ of the analyzed participants of the cholesterol-lowering drug collective. Further analyzed drug-gene combinations (Table 3 ) mentioned by CPIC, but with lower levels of evidence, e.g., related to CYP3A4/5, are listed in Supplement 2. 
Table 2. Number and percentage of participants within the study population and study collectives with prescriptions of medication with functional impact on the cytochromes, catalytic subunit, and transporters under study within the baseline year.

\begin{tabular}{|c|c|c|c|}
\hline Collective & Gene & $\begin{array}{l}\text { Participants with } \\
\text { inhibitor or } \\
\text { antagonist } \\
\text { prescription }\end{array}$ & $\begin{array}{l}\text { Percentage } \\
\text { within collective }\end{array}$ \\
\hline \multirow{7}{*}{$\begin{array}{l}\text { Complete study } \\
\text { population }\end{array}$} & СYР2C9 & 4058 & 37.76 \\
\hline & СYP2C19 & 3677 & 34.21 \\
\hline & VKORC1 & 110 & 1.02 \\
\hline & SLCO1B1 & 6327 & 58.87 \\
\hline & $A B C B 1$ & 7589 & 70.61 \\
\hline & СУРЗА4 & 5416 & 50.39 \\
\hline & СУРЗА5 & 35 & 0.33 \\
\hline \multirow{7}{*}{$\begin{array}{l}\text { Anticoagulant/ } \\
\text { antiplatelet }\end{array}$} & СYP2C9 & 3437 & 41.35 \\
\hline & CYP2C19 & 2913 & 35.04 \\
\hline & VKORC1 & 0 & 0.00 \\
\hline & SLCO1B1 & 5071 & 61.00 \\
\hline & $A B C B 1$ & 5999 & 72.16 \\
\hline & СУРЗА4 & 4232 & 50.91 \\
\hline & СYРЗА5 & 23 & 0.28 \\
\hline \multirow{7}{*}{$\begin{array}{l}\text { Cholesterol- } \\
\text { lowering drug }\end{array}$} & CYP2C9 & 418 & 21.84 \\
\hline & CYP2C19 & 542 & 28.32 \\
\hline & VKORC1 & 74 & 3.87 \\
\hline & SLCO1B1 & 931 & 48.64 \\
\hline & $A B C B 1$ & 1222 & 63.85 \\
\hline & СYР3А4 & 893 & 46.66 \\
\hline & СУРЗА5 & 9 & 0.47 \\
\hline \multirow[t]{7}{*}{ Y57.9! } & СYP2C9 & 203 & 38.96 \\
\hline & СYP2C19 & 222 & 42.61 \\
\hline & VKORC1 & 36 & 6.91 \\
\hline & SLCO1B1 & 325 & 62.38 \\
\hline & $A B C B 1$ & 368 & 70.63 \\
\hline & СУР3А4 & 291 & 55.85 \\
\hline & СУРЗАБ & 3 & 0.58 \\
\hline
\end{tabular}

Antagonists and inhibitors were identified according to respective entries on DrugBank Online, a database for drug and drug target information.

\section{DISCUSSION}

For some cardiovascular drugs such as clopidogrel, warfarin, and simvastatin, robust data on clinical benefits due to preemptive pharmacogenetic testing provide a promising outlook for clinical implementation in routine care [3]. However, for guided therapy with most cardiovascular drugs, cost-effectiveness of pharmacogenetic testing in German routine care is not sufficiently elucidated yet. Therefore, the EMPAR study evaluates the genetic variability on the basis of participants' metabolic profiles with regard to cardiovascular drugs and will consider pharmacoepidemiological evaluations concerning ADRs and pharmacoeconomic evaluations on the utilization and costs of health insurance services according to healthcare claims records in German routine care in future analyses. For this first evaluation, datasets of 10,748 participants were analyzed for basic population characteristics, DNA-quality control parameters and prevalence of pharmacogenetic variants which could be risk factors for ADRs.

In our study population, a tendency of an increased quantity of diagnoses and prescription entries in elderly participants compared to the young and moderate age participants was observed already at baseline (Supplement 3). This observation is concordant with studies evaluating age-specific trends such as comorbidity and polypharmacy [24-27]. A high percentage of detected prescriptions within the individual baseline year included inhibitors and antagonists of the cytochromes, the catalytic subunit, and transporters under study (Table 2). Especially here, phenotype prediction of the metabolizer status with regard to the affected medication of interest and the evaluation of ADRs could be compromised due to phenoconversion. Affected participants may have a higher risk of drug-drug, e.g., drug-drug-gene interactions concerning the medication of study interest $[28,29]$. Furthermore, $21 \%$ of participants receiving $\geq 2$ and especially $2.3 \%$ identified with $\geq 5$ prescriptions in each quarter of the baseline year may be at higher risk of such interactions [30]. A previous study in the Netherlands concerning long-term polypharmacy ( $>240$ days over a year) showed similar percentages of participants with long-term use of $\geq 2$ drugs ( $20 \%$ elderly participants) and of more than 5 drugs (4\% elderly participants) [31].

Therapeutic treatment with cardiovascular drugs in order to prevent coronary morbidity and mortality should also consider a risk reduction of ADRs [32]. Genetic risk factors can predict the
A

\begin{tabular}{|c|c|c|c|c|c|}
\hline Gene & Variant & WW & MW & MM & NA \\
\hline \multirow[t]{7}{*}{ CYP2C9 } & rs1057910 & 9212 & 1384 & 47 & 105 \\
\hline & rs1799853 & 8174 & 2315 & 174 & 85 \\
\hline & rs28371685 & 10565 & 56 & 0 & 127 \\
\hline & rs28371686 & 10647 & 1 & 0 & 100 \\
\hline & rs56165452 & 10584 & 0 & 164 & 0 \\
\hline & rs7900194 & 10669 & 3 & 0 & 76 \\
\hline & rs9332131 & 10313 & 0 & 435 & 0 \\
\hline \multirow[t]{4}{*}{ CYP2C19 } & rs12248560 & 6386 & 3662 & 555 & 145 \\
\hline & rs41291556 & 10556 & 63 & 0 & 129 \\
\hline & rs4244285 & 7514 & 2575 & 274 & 385 \\
\hline & rs4986893 & 10661 & 7 & 0 & 80 \\
\hline SLCO1B1 & rs4149056 & 7274 & 3090 & 304 & 80 \\
\hline VKORC1 & rs9923231 & 3916 & 5077 & 1644 & 111 \\
\hline
\end{tabular}

B

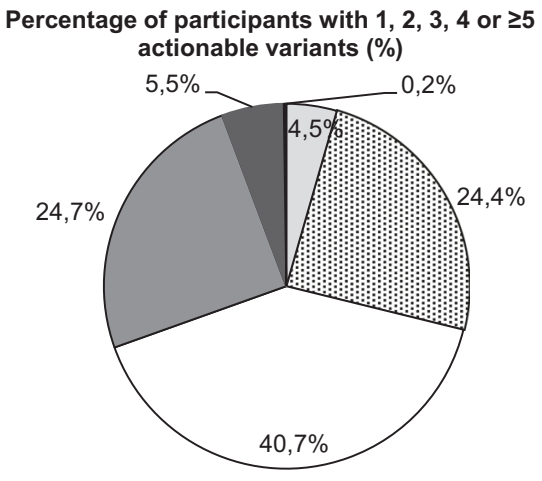

口0 回1 $12 \square 3 \square 4 \square \geq 5$

Fig. 5 Relevant actionable variants in the EMPAR population. A Number of carriers of pharmacogenetic markers with PharmGKB Evidence level 1A/1B with regard to cardiovascular indications. Major allele here defined as wild type (W); M: minor allele or actionable variant respectively; NA: information not available; MW: actionable variant/wild type. B Proportions of participants in the complete study population with $1,2,3,4$, and $\geq 5$ actionable variants shown in $\mathbf{A}$. 
Table 3. Proportion of participants with relevant prescriptions (main medication) and extreme phenotypes of the evaluated cytochromes, catalytic subunit, and transporters in the cardiovascular drug collectives.

\begin{tabular}{|c|c|c|c|c|c|}
\hline Collective & Prescription & Extreme phenotype (predicted) & Gene & Participants affected & Percentage (within collective) \\
\hline \multirow[t]{46}{*}{$\mathrm{A} / \mathrm{T}$} & Apixaban & $\mathrm{PF}$ & $A B C B 1$ & 229 & 2.8 \\
\hline & Apixaban & PF & VKORC1 & 143 & 1.7 \\
\hline & Apixaban & PM & CYP2C9 & 38 & 0.5 \\
\hline & Apixaban & PM & CYP2C19 & 23 & 0.3 \\
\hline & Apixaban & UM & CYP2C19 & 59 & 0.7 \\
\hline & Clopidogrel & PF & $A B C B 1$ & 523 & 6.3 \\
\hline & Clopidogrel & PF & VKORC1 & 333 & 4.0 \\
\hline & Clopidogrel & PM & CYP2C9 & 96 & 1.2 \\
\hline & Clopidogrel & PM & CYP2C19 & 56 & 0.7 \\
\hline & Clopidogrel & UM & CYP2C19 & 113 & 1.4 \\
\hline & Clopidogrel+ASS & PF & $A B C B 1$ & 10 & 0.1 \\
\hline & Clopidogrel+ASS & $\mathrm{PF}$ & VKORC1 & 10 & 0.1 \\
\hline & Clopidogrel+ASS & PM & CYP2C9 & 2 & 0.0 \\
\hline & Clopidogrel+ASS & PM & CYP2C19 & 1 & 0.0 \\
\hline & Clopidogrel+ASS & UM & CYP2C19 & 5 & 0.1 \\
\hline & Dabigatranetexilat & PF & $A B C B 1$ & 63 & 0.8 \\
\hline & Dabigatranetexilat & $\mathrm{PF}$ & VKORC1 & 37 & 0.4 \\
\hline & Dabigatranetexilat & PM & CYP2C19 & 7 & 0.1 \\
\hline & Dabigatranetexilat & PM & CYP2C9 & 7 & 0.1 \\
\hline & Dabigatranetexilat & UM & CYP2C19 & 15 & 0.2 \\
\hline & Edoxaban & PF & $A B C B 1$ & 40 & 0.5 \\
\hline & Edoxaban & PF & VKORC1 & 29 & 0.3 \\
\hline & Edoxaban & PM & CYP2C9 & 7 & 0.1 \\
\hline & Edoxaban & PM & CYP2C19 & 3 & 0.0 \\
\hline & Edoxaban & UM & CYP2C19 & 12 & 0.1 \\
\hline & Phenprocoumon & PF & $A B C B 1$ & 315 & 3.8 \\
\hline & Phenprocoumon & PF & VKORC1 & 215 & 2.6 \\
\hline & Phenprocoumon & PM & CYP2C9 & 47 & 0.6 \\
\hline & Phenprocoumon & PM & CYP2C19 & 30 & 0.4 \\
\hline & Phenprocoumon & UM & CYP2C19 & 63 & 0.8 \\
\hline & Prasugrel & PF & $A B C B 1$ & 103 & 1.2 \\
\hline & Prasugrel & PF & VKORC1 & 89 & 11 \\
\hline & Prasugrel & PM & CYP2C9 & 17 & 0.2 \\
\hline & Prasugrel & PM & CYP2C19 & 8 & 0.1 \\
\hline & Prasugrel & UM & CYP2C19 & 29 & 0.3 \\
\hline & Rivaroxaban & PF & $A B C B 1$ & 523 & 6.3 \\
\hline & Rivaroxaban & PF & VKORC1 & 369 & 4.4 \\
\hline & Rivaroxaban & PM & CYP2C9 & 97 & 1.2 \\
\hline & Rivaroxaban & PM & CYP2C19 & 70 & 0.8 \\
\hline & Rivaroxaban & UM & CYP2C19 & 109 & 1.3 \\
\hline & Ticagrelor & PF & $A B C B 1$ & 148 & 1.8 \\
\hline & Ticagrelor & PF & VKORC1 & 79 & 1.0 \\
\hline & Ticagrelor & PM & CYP2C9 & 29 & 0.3 \\
\hline & Ticagrelor & PM & CYP2C19 & 17 & 0.2 \\
\hline & Ticagrelor & UM & CYP2C19 & 31 & 0.4 \\
\hline & Warfarin & PM & CYP2C19 & 1 & 0.0 \\
\hline \multirow[t]{4}{*}{ CLD } & Atorvastatin & PM & СУРЗА5 & 827 & 43.2 \\
\hline & Atorvastatin & PF & $A B C B 1$ & 200 & 10.4 \\
\hline & Atorvastatin & PF & SLCO1B1 & 22 & 1.1 \\
\hline & Fluvastatin & PM & СУР $3 A 5$ & 18 & 0.9 \\
\hline
\end{tabular}


Table 3. continued

\begin{tabular}{|c|c|c|c|c|c|}
\hline \multirow[t]{9}{*}{ Collective } & Prescription & Extreme phenotype (predicted) & Gene & Participants affected & Percentage (within collective) \\
\hline & Fluvastatin & PF & $A B C B 1$ & 7 & 0.4 \\
\hline & Lovastatin & PM & CYP3A5 & 1 & 0.1 \\
\hline & Pravastatin & PM & СУРЗА5 & 35 & 1.8 \\
\hline & Pravastatin & $\mathrm{PF}$ & $A B C B 1$ & 8 & 0.4 \\
\hline & Pravastatin & $\mathrm{PF}$ & SLCO1B1 & 2 & 0.1 \\
\hline & Rosuvastatin & PM & CYP3A5 & 3 & 0.2 \\
\hline & Simvastatin & $\mathrm{PF}$ & SLCO1B1 & 16 & 0.8 \\
\hline & Simvastatin & PM & СYР3A4 & 4 & 0.2 \\
\hline
\end{tabular}

$A / T$ anticoagulant/antiplatelet, CLD cholesterol-lowering drug, ASS acetylsalicylic acid, $P F$ poor function, $P M$ poor metabolizer, UM ultrarapid metabolizer.

individual response to particular drugs and the risk of ADRs to some extent. Around $95.5 \%$ of all participants are carrier of at least one relevant PGx variant (PharmGKB clinical annotation Evidence level $1 \mathrm{~A}, \mathrm{~B})$. Thus, these data are comparable to other studies, for instance, reported by the eMERGE-PGx program where more than $96 \%$ of samples displayed relevant actionable PGx variants [33]. Furthermore, the Mayo RIGHT program showed that $99 \%$ of participants were carrier of at least one of such variants including also CYP2D6 variants and $3 \%$ of participants even were carrier of actionable PGx variants in CYP2D6, CYP2C9, CYP2C19, SLCO1B1, and VKORC1 [3]. The analysis of genotype data of 11 genes of 44,000 participants of the Estonian biobank revealed that $99.8 \%$ of genotypes were associated with an increased risk profile with regard to at least one medication [34]. Thus, our study provided additional evidence that pharmacogenetic risk factors for ADRs are prevalent in a majority of representatives of an expected primarily Caucasian population sample.

Previous studies predicting metabolizer phenotypes in Caucasian populations reported that, e.g., in a Croatian population $3.98 \%$ of study participants were PMs for CYP2C9, while in a small Italian study population, $1.7 \%$ were PM due to homozygosity for the CYP2C19*2 variant $[35,36]$. According to the PharmGKB CYP2C9 Frequency Table, a prevalence of $2.6 \%$ in a European population is estimated for a PM phenotype [37]. However, in the anticoagulant collective of the EMPAR study, a higher phenotype frequency was observed. Here $4 \%$ of participants presented with a CYP2C9 PM phenotype. Phenotype frequency information was not provided by PharmGKB for VKORC1 and is rarely reported in studies for Caucasian populations in Europe. In the EMPAR anticoagulant/ antiplatelet collective $15.3 \%$ of study participants were predicted with a deficient VKORC1 phenotype due to homozygosity for the VKORC1 rs9923231 TT variant. However, a lower frequency was reported by Schelleman et al. (Supplement 1 - Fig. 5) [38]. An evaluation of CYP2C19 predicted phenotypes concerning ethnic groups and the geographic regions across world populations showed that CYP2C19 PM phenotypes differed in Caucasian populations from $1.56 \%$ to $3.61 \%$. Thereby, populations from Europe were represented with the lowest frequency of about $1.76 \%$ [39]. According to the PharmGKB CYP2C19 Frequency Table, CYP2C19 PMs are estimated in $2.3 \%$ of a European population [40]. Similar to these findings, PMs for CYP2C19 were detected in $2.6 \%$ of the EMPAR study population. The high concordance of our results with data from PharmGKB and data from literature concerning the distribution of phenotypes showed that the potential selection bias in this study due to many insurants that were excluded [16], mainly did not affect the phenotype frequencies predicted in this study.

CYP2C9 poor metabolism or VKORC1 poor enzymatic activity is associated with a higher risk of bleeding events upon treatment with several coumarins that were of primary study interest. Furthermore, in previous studies, CYP2C19 PMs were shown to be at higher risk of adverse cardiovascular events in treatment with clopidogrel [41]. In our study population, such phenotypes were present also in parallel with prescriptions of the respective cardiovascular drugs and could have increased the risk of ADRs for these participants.

Among drug-gene pairs relevant for the cholesterol-lowering drug cohort, for simvastatin-SLCO1B1, a risk of ADRs such as muscle problems including myalgias and rhabdomyolysis was reported in previous studies for the poor metabolism/function phenotype $[42,43]$. However, relevant genes involved in statin transport or metabolism such as SLCO1B1 (2.1.\%) or CYP3A4 (0.2\%) occur in the cholesterol-lowering drug (statin) collective only as small fractions with that phenotype.

In future analyses, the impact of actionable variants, extreme phenotypes, and metabolic profiles on ADRs and cost expenditures in routine care will be investigated considering also low evidence level drug-gene pairs. Furthermore, concomitant medication and relevant associated pharmacogenes such as CYP2D6 will be of additional interest concerning drug-drug-gene e interactions. Screening for new relevant SNPs of pharmacogenes associated with ADRs will be performed especially considering the Y57.9!-diagnosis collective.

\section{CONCLUSION}

Genetic analysis considering healthcare claims records provides valuable information to assess the risk of ADRs. Preemptive risk assessment probably gets even more important to elderly, comorbid patients under long-term polypharmaceutical therapies as identified in about $2.3 \%$ of the EMPAR study participants. On marker level, a majority of study participants were carrier of at least one actionable variant concerning pharmacogenes relevant for cardiovascular drugs. Furthermore, on metabolic profile level, important drug-gene combinations that could affect the safety of drug therapy were observed. With these and further evaluations, we aim to contribute to the accumulation of evidence regarding the clinical utility of pharmacogenetic markers to improve the information basis for reimbursement policy and adoption of preemptive testing into routine care in Germany.

\section{REFERENCES}

1. Meier F, Maas R, Sonst A, Patapovas A, Müller F, Plank-Kiegele B, et al. Adverse drug events in patients admitted to an emergency department: an analysis of direct costs. Pharmacoepidemiol Drug Saf. 2015;24:176-86.

2. Leendertse AJ, Egberts $A C$, Stoker $L$, van den Bemt PM. Frequency of and risk factors for preventable medication-related hospital admissions in the Netherlands. Arch Intern Med. 2008;168:1890-6.

3. Ji Y, Skierka JM, Blommel JH, Moore BE, VanCuyk DL, Bruflat JK, et al. Preemptive pharmacogenomic testing for precision medicine: a comprehensive analysis of five actionable pharmacogenomic genes using next-generation DNA sequencing and a customized CYP2D6 genotyping cascade. J Mol Diagn. 2016;18:438-45. 
4. Unternehmensdaten | Die Techniker - Presse und Politik. 2021 https://www.tk.de/ presse/tk-unternehmensdaten-2051018. Accessed 20 July 2020.

5. Ingelman-Sundberg M. Human drug metabolising cytochrome P450 enzymes: prop erties and polymorphisms. Naunyn Schmiedebergs Arch Pharm. 2004;369:89-104.

6. Dean L. Warfarin therapy and VKORC1 and CYP genotype. Medical genetics summaries [Internet]; 2012.

7. Hoenig MR, Walker PJ, Gurnsey C, Beadle K, Johnson L. The C3435T polymorphism in $A B C B 1$ influences atorvastatin efficacy and muscle symptoms in a high-risk vascular cohort. J Clin Lipido. 2011;5:91-6.

8. Voora D, Shah SH, Spasojevic I, Ali S, Reed CR, Salisbury BA, et al. The SLCO1B1*5 genetic variant is associated with statin-induced side effects. J Am Coll Cardiol. 2009;54:1609-16.

9. Pasanen MK, Neuvonen M, Neuvonen PJ, Niemi M. SLCO1B1 polymorphism markedly affects the pharmacokinetics of simvastatin acid. Pharmacogenet Genomics. 2006;16:873-9.

10. Sherry ST, Ward MH, Kholodov M, Baker J, Phan L, Smigielski EM, et al. dbSNP: the NCBI database of genetic variation. Nucleic Acids Res. 2001;29:308-11.

11. Gaedigk A, Ingelman-Sundberg M, Miller NA, Leeder JS, Whirl-Carrillo M, Klein TE. The Pharmacogene Variation (PharmVar) Consortium: incorporation of the Human Cytochrome P450 (CYP) Allele Nomenclature Database. Clin Pharm Ther 2018;103:399-401.

12. Thorn CF, Klein TE, Altman RB. PharmGKB: the Pharmacogenomics Knowledge Base. Methods Mol Biol. 2013;1015:311-20.

13. PharmGKB. What is PharmGKB?: PharmGKB. https://www.pharmgkb.org/ whatlsPharmgkb. Accessed 20 July 2020.

14. Hočevar K, Maver A, Peterlin B. Actionable pharmacogenetic variation in the Slovenian genomic database. Front Pharm. 2019;10:240.

15. Huebner T, Steffens M, Linder R, Fracowiak J, Langner D, Garling M, et al. Influence of metabolic profiles on the safety of drug therapy in routine care in Germany: protocol of the cohort study EMPAR. BMJ Open. 2020;10:e032624.

16. Devices FIfDaM. Influence of metabolic profiles on drug safety in routine care German Clinical Trials Register: DRKS - German Clinical Trials Register. https://www. drks.de/drks web/navigate.do?navigationld=trial.HTML\&TRIAL ID=DRKS00013909. Accessed 21 July 2020.

17. Jensen L, Børsting C, Dalhoff K, Morling N. Evaluation of the iPLEX ${ }^{\circledR}$ ADME PGx Pro Panel and allele frequencies of pharmacogenetic markers in Danes. Clin Biochem. 2016;49:1299-301.

18. Bioscience A. Agena-Bioscience-VeriDose-Core-Variant-List-GEN001802.pdf. https:// www.agenabio.com/products/panel/veridose-core-panel/. Accessed 17 May 2021.

19. Purcell S, Neale B, Todd-Brown K, Thomas L, Ferreira MAR, Bender D, et al. PLINK: a tool set for whole-genome association and population-based linkage analyses. Am J Hum Genet. 2007;81:559-75.

20. Gasparini A. comorbidity: an R package for computing comorbidity scores J Open Source Softw. 2018;3:648

21. Caudle KE, Sangkuhl K, Whirl-Carrillo M, Swen JJ, Haidar CE, Klein TE, et al. Standardizing CYP2D6 genotype to phenotype translation: consensus recommendations from the Clinical Pharmacogenetics Implementation Consortium and Dutch Pharmacogenetics Working Group. Clin Transl Sci. 2020;13:116-24.

22. Kjeldsen SE. Hypertension and cardiovascular risk: general aspects. Pharm Res. 2018;129:95-9.

23. Whirl-Carrillo M, McDonagh EM, Hebert JM, Gong L, Sangkuhl K, Thorn CF, et al. Pharmacogenomics knowledge for personalized medicine. Clin Pharm Ther. 2012;92:414-7.

24. Modig K, Andersson T, Drefahl S, Ahlbom A. Age-specific trends in morbidity, mortality and case-fatality from cardiovascular disease, myocardial infarction and stroke in advanced age: evaluation in the Swedish population. PLoS One. 2013;8:e64928.

25. Yazdanyar A, Newman AB. The burden of cardiovascular disease in the elderly: morbidity, mortality, and costs. Clin Geriatr Med. 2009;25:563-77.

26. van den Akker M, Vaes B, Goderis G, Van Pottelbergh G, De Burghgraeve T, Henrard S. Trends in multimorbidity and polypharmacy in the Flemish-Belgian population between 2000 and 2015. PLoS One. 2019;14:e0212046.

27. Zhang N, Sundquist J, Sundquist $\mathrm{K}$, Ji J. An increasing trend in the prevalence of polypharmacy in Sweden: a nationwide register-based study. Front Pharm. 2020;11:326.

28. Hahn M, Roll SC. The influence of pharmacogenetics on the clinical relevance of pharmacokinetic drug-drug interactions: drug-gene, drug-gene-gene and drugdrug-gene interactions. Pharmaceuticals (Basel). 2021;14:487.

29. Klomp SD, Manson ML, Guchelaar HJ, Swen JJ. Phenoconversion of cytochrome P450 metabolism: a systematic review. J Clin Med. 2020;9:2890.

30. Maher RL, Hanlon J, Hajjar ER. Clinical consequences of polypharmacy in elderly. Expert Opin Drug Saf. 2014;13:57-65.

31. Veehof L, Stewart R, Haaijer-Ruskamp F, Jong BM. The development of polypharmacy. A longitudinal study. Fam Pract. 2000;17:261-7.
32. Peters BJ, Klungel $\mathrm{OH}$, de Boer A, Ch Stricker BH, Maitland-van der Zee $\mathrm{AH}$. Pharmacogenetics of cardiovascular drug therapy. Clin Cases Min Bone Metab. 2009;6:55-65.

33. Bush WS, Crosslin DR, Owusu-Obeng A, Wallace J, Almoguera B, Basford MA, et al. Genetic variation among 82 pharmacogenes: the PGRNseq data from the eMERGE Network. Clin Pharmacol Ther. 2016;100:160-9.

34. Reisberg S, Krebs K, Lepamets $M$, Kals M, Mägi R, Metsalu K, et al. Translating genotype data of 44,000 biobank participants into clinical pharmacogenetic recommendations: challenges and solutions. Genet Med. 2019;21:1345-54.

35. Ganoci L, Božina T, Mirošević Skvrce N, Lovrić M, Mas $P$, Božina N. Genetic polymorphisms of cytochrome P450 enzymes: CYP2C9, CYP2C19, CYP2D6, CYP3A4, and CYP3A5 in the Croatian population. Drug Metab Pers Ther. 2017;32:11-21.

36. Scordo MG, Caputi AP, D'Arrigo C, Fava G, Spina E. Allele and genotype frequencies of CYP2C9, CYP2C19 and CYP2D6 in an Italian population. Pharm Res. 2004;50:195-200.

37. PharmGKB. CYP2C9 Frequency Table: PharmGKB. https://www.pharmgkb.org/ page/cyp2c9RefMaterials. Acessed 20 May 2021.

38. Schelleman H, Chen Z, Kealey C, Whitehead AS, Christie J, Price M, et al. Warfarin response and vitamin $\mathrm{K}$ epoxide reductase complex 1 in African Americans and Caucasians. Clin Pharm Ther. 2007;81:742-7.

39. Fricke-Galindo I, Céspedes-Garro C, Rodrigues-Soares F, Naranjo MEG, Delgado Á, de Andrés $\mathrm{F}$, et al. Interethnic variation of CYP2C19 alleles, 'predicted' phenotypes and 'measured' metabolic phenotypes across world populations. Pharmacogenomics J. 2016;16:113-23.

40. PharmGKB. CYP2C19 Frequency Table.

41. Scott SA, Sangkuhl K, Stein CM, Hulot JS, Mega JL, Roden DM, et al. Clinical Pharmacogenetics Implementation Consortium guidelines for CYP2C19 genotype and clopidogrel therapy: 2013 update. Clin Pharm Ther. 2013;94:317-23.

42. Xiang Q, Chen S-Q, Ma L-Y, Hu K, Zhang Z, Mu G-Y, et al. Association between SLCO1B1 T521C polymorphism and risk of statin-induced myopathy: a metaanalysis. Pharmacogenomics J. 2018;18:721-9.

43. Ramsey LB, Johnson SG, Caudle KE, Haidar CE, Voora D, Wilke RA, et al. The clinical pharmacogenetics implementation consortium guideline for SLCO1B1 and simvastatin-induced myopathy: 2014 update. Clin Pharm Ther. 2014;96:423-8.

\section{AUTHOR CONTRIBUTIONS}

$\mathrm{JS}, \mathrm{BH}, \mathrm{MS}$, and $\mathrm{RL}$ were responsible for study design and funding acquisition. $\mathrm{DL}, \mathrm{US}$, and FF coordinated the selection of participants and managed recruitment and data transfer from the Techniker Krankenkasse. Draft conceptualization by TH. JF, MS, and SH designed the analyses for the draft. JF and MS performed the analyses. JF and TH prepared the original draft. $C S, J S, B H, R L, C R, S H$, and $M S$ critically reviewed and edited the draft. All authors revised the manuscript and approved the final version.

\section{FUNDING}

This research was funded by the Innovation Fund of the Gemeinsamer Bundesausschuss (Federal Joint Committee), grant number: 01VSF16047. Open Access funding enabled and organized by Projekt DEAL.

\section{COMPETING INTERESTS}

The authors declare no competing interests.

\section{ETHICS APPROVAL}

The study protocol was approved by the Ethics Committee of the Medical Faculty at the University of Bonn (339/17) and written informed consent was provided by all study participants.

\section{ADDITIONAL INFORMATION}

Supplementary information The online version contains supplementary material available at https://doi.org/10.1038/s41397-022-00268-6.

Correspondence and requests for materials should be addressed to Tatjana Huebner.

Reprints and permission information is available at http://www.nature.com/reprints

Publisher's note Springer Nature remains neutral with regard to jurisdictional claims in published maps and institutional affiliations. 
Open Access This article is licensed under a Creative Commons Attribution 4.0 International License, which permits use, sharing, adaptation, distribution and reproduction in any medium or format, as long as you give appropriate credit to the original author(s) and the source, provide a link to the Creative Commons license, and indicate if changes were made. The images or other third party material in this article are included in the article's Creative Commons license, unless indicated otherwise in a credit line to the material. If material is not included in the article's Creative Commons license and your intended use is not permitted by statutory regulation or exceeds the permitted use, you will need to obtain permission directly from the copyright holder. To view a copy of this license, visit http://creativecommons. org/licenses/by/4.0/.

(c) The Author(s) 2022 